\title{
A SYSTEM OF FORTRAN FOR THREE-DIMENSIONAL TEXTURE ANALYSIS
}

\author{
J. JURA, J. POSPIECH, and H. J. BUNGE \\ Polish Academy of Sciences, Institute of Metallurgy, Craców, Poland, and Academy of Sciences \\ of the GDR, Institute of Solid State Physics and Materials Research, Dresden, GDR.
}

(Received November 27, 1973)

\begin{abstract}
A system of FORTRAN programmes is being described which allows most of the standard problems of threedimensional texture analysis to be easily carried out. It requires about $25 \mathrm{~K}$ memory space and it may easily be enlarged by incorporating further subroutines.
\end{abstract}

\section{INTRODUCTION}

In the last few years three-dimensional texture analysis has developed into an efficient tool, which is employed in an increasing number of investigations. Its applicability is not only confined to metals and alloys. The method has also been applied to polymers, ceramic materials, and geological samples.

The fundamental problem in texture analysis is to calculate the three-dimensional orientation distribution function, which cannot be measured directly from experimental data mostly pole figures. Besides this, however, it is often necessary to calculate other quantities, such as the inverse pole figures, or normal pole figures for certain directions which cannot be measured directly, or macroscopic anisotropy of physical properties, for example, the elastic, plastic, or magnetic anisotropy. All these require rather lengthy numerical calculations which can only be carried out by a computer. Thus, for the method to be applicable as a routine approach, it is necessary to have a comprehensive system of computer programmes which allow the different calculations to be easily carried out, and which is sufficiently versatile to meet the specific requirements of many kinds of problems.

Computer programmes for three-dimensional texture analysis have been developed and applied in a number of laboratories and have been reported in various publications. ${ }^{1-4}$ As to our knowledge, however, a full length description of a comprehensive system of programmes has not yet been published. This may be due to the rapid development of the methods of texture analysis themselves as well as of the computer technique. This development is certainly not yet finished. Nevertheless, we feel that a certain state might have been reached at which the publication of a system of routine programmes might be useful. The programmes we are going to describe here are written in FORTRAN. They require about $25 \mathrm{~K}$ memory space and include subroutines for most of the standard problems thus far encountered in three-dimensional texture analysis for cubic materials and orthorhombic (or higher) specimen symmetry (sheet symmetry). Furthermore, the system may easily be enlarged by incorporating further subroutines. In the following we shall give a brief outline of the system. A full length description including the full FORTRAN text is being given elsewhere. ${ }^{5}$

\section{THE SYSTEM OF PROGRAMMES}

The system consists of three parts:

1) The library of subroutines, which perform the different mathematical operations;

2) the master programmes, which invoke the different subroutines out of the library and put them together according to each specific problem; and

3) the library of the tables of constants.

The library of subroutines is the main part of the system. It determines what calculations can be carried out at all. The subroutines are stored on a magnetic tape from which they are called in by their names according to the scheme of calculations determined by the master programme. In the present 
state the library contains (besides some minor ones) the following main subroutines:

\section{COREC}

This programme carries out the absorption and other corrections of experimental pole figure data. It multiplies the experimental values by factors arbitrarily chosen for each value of the angular coordinate $\Phi$. Furthermore, it carries out a least squares fit of transmission and back-reflection data. The output of this programme are corrected (not normalized) pole figure values.

\section{TWODIM}

This programme develops the pole figure into a series of spherical harmonics of orthorhombic symmetry (sheet symmetry). It accepts corrected (not necessarily normalized) pole figure values in angular steps $\Delta \Phi, \Delta \beta$ (integer parts of $90^{\circ}$ ). Standard values are $\Delta \Phi=\Delta \beta=5^{\circ}$. Its output is the normalizing factor, the normalized pole figure, and the table of coefficients $F_{l}^{v}\left(h_{i}\right)_{\text {exp }}$ (For the definitions see ref. 6). In this programme, as well as in the following ones, the series development may be extended up to a maximum value of $l_{\max }=34$.

\section{COEF}

This programme accepts $F_{l}^{v}\left(h_{i}\right)_{\exp }$ values of three or four pole figures. It calculates the coefficients $C_{l}^{\mu \nu}$, their variance $\delta\left(C_{l}^{\mu \nu}\right)$, the variance of the $F$ values $\delta\left(F_{l}^{v}\right)$ as well as mean values of these quantities over $\mu$ and $\nu$ and the texture index $\mathrm{J}$. The coefficients $C_{l}^{\mu \nu}$, being the fundamental quantities in texture analysis, are given out on paper tape along with certain information characterizing the specimen so that they are available for any further calculation that may become necessary later on. Finally in this programme also a set of pole figure coefficients $F_{l}^{v}\left(h_{i}\right)_{\text {cal }}$ is being recalculated from the coefficients $C_{l}^{\mu \nu}$.

\section{POLO}

This programme accepts the coefficients $F_{l}^{v}\left(h_{i}\right)_{\text {cal }}$ and recalculates theoretical pole figure values for those pole figures which were used as input in the programme COEF.

\section{POLV}

This programme calculates coefficients $F_{l}^{\nu}\left(h_{i}\right)_{\text {cal }}$ and pole figure values for any pole figure out of the set (100), (110), (111), (102), (112), (122), (103), (113). Its input are the coefficients $C_{l}^{\mu \nu}$. In this programme and the previous one angular steps $\Delta \Phi=\Delta \beta=5^{\circ}$ are used.

\section{INVA}

This programme accepts the coefficients $C_{l}^{\mu \nu}$ and calculates the coefficients $H_{l}^{\mu}$ of an inverse pole figure as well as the inverse pole figure itself. It can be applied to rolling, normal and transverse directions or any arbitrarily chosen direction determined by angular coordinates $\gamma, \delta$. The inverse pole figure values are calculated in standard angular steps $\Delta \Phi=\Delta \beta=2.5^{\circ}$.

\section{UNKC}

In this programme the three-dimensional orientation distribution function is calculated from coefficients $C_{l}^{\mu \nu}$. It can be used in two versions which print const. $-\varphi_{1}$ or const. $-\varphi_{2}$ tables respectively. The angular steps are fixed $\Delta \varphi_{1}=\Delta \Phi=\Delta \varphi_{2}=$ $5^{\circ}$. This part of the calculations is the most time consuming one. In the standard version it takes several minutes.

Besides these main subroutines some other ones are available according to certain special problems. For example, if pole figures are available only in the back-reflection region the coefficients $C_{l}^{\mu \nu}$ can be calculated directly by a least squares method from the pole figure values without making use of the pole figure coefficients $F_{l}^{\nu}$. (This method needs more memory space and its execution time is much longer than in the standard method). Or if different pole figures are superimposed because of identical or nearly identical $\theta$-values they may be artificially separated by a specific programme. Sometimes it may be convenient to represent the three-dimensional orientation distribution function in other orientation coordinates than the Euler angles, for example, the rotation axis and angle. This can be achieved by a further special subroutine. Or finally, orientation mean values of anisotropic properties may be calculated from $C_{l}^{\mu \nu}$-values according to an appropriate model, for example, the plastic anisotropy according to the Taylor- 
model. The library of subroutines is so designed such that it can be easily enlarged by adding further programmes, which carry out other operations.

The master programmes are rather short organizing programmes which fit the subroutines together and determine the way they are to be run through.

The library of constants contains the following quantities which are used by the subroutines. (For the definitions, see ref. 6 ).

1) $\bar{P}_{l}^{n}(\Phi)=\sum_{s=0}^{l} a_{l}^{\prime n s} \cos s \Phi \quad \Phi=0^{\circ}\left(5^{\circ}\right) 90^{\circ}$

2) $\dot{\dot{k}} \mu\left(h_{i}\right)$

$$
\begin{gathered}
h_{i}=(100,110,111, \\
102,112,122, \\
103,113)
\end{gathered}
$$

3) $a_{l}^{\prime n s} \quad l, n, s$ even

4) $\dot{B}_{l}^{m \mu} \quad m=0$ (4) $l$

5) $a_{l}^{\prime m n s}$

(for certain values of $l, m, n, s)$

Presently all these quantities are stored up to $l_{\max }=34$. Hence all calculations can be carried out up to this degree which proved sufficient in most problems encountered thus far.

The system of programmes described above allows all the standard calculations in threedimensional texture analysis, as well as some special ones, to be easily carried out at a sufficient degree of accuracy. It is written in a language which is understood by different types of computers, and it is easy to use for routine work. A full length description, which should enable anybody to apply the above system, is being given elsewhere. $^{5}$

\section{REFERENCES}

1. H. J. Bunge and H. Sandmann, Mber. Dtsch. Akad. Wiss. 5, 344 (1963).

2. H. J. Bunge, Kristall und Technik. 6, 429 (1971).

3. J. Jura, Diploma Thesis AGH Kraków (1969).

4. J. Pospiech, Mat. Konf. Sprawozd. PAN Krynica (1969).

5. J. Jura, J. Pospiech and H. J. Bunge, Freiberger Forschungshefte, to be published.

6. H. J. Bunge, Mathematische Methoden der Texturanalyse (Akademieverlag Berlin 1969). 